\title{
SentiALG: Automated Corpus Annotation for Algerian Sentiment Analysis
}

\author{
Imane Guellil ${ }^{1,2}$, Ahsan Adeel $^{3}$, Faical Azouaou ${ }^{2}$, and Amir Hussain ${ }^{3}$ \\ 1 Ecole Superieure des sciences appliquées d'Alger ESSA-alger \\ i.guellil@essa-alger.dz \\ 2 Laboratoire des Méthodes de Conception des Systèmes (LMCS), Ecole nationale \\ Supérieure d'Informatique,BP 68M, 16309, Oued-Smar, Alger, Algérie \\ i_guellil@esi.dz, f_azouaou@esi.dz \\ 3 Institute of Computing science and Mathematics, School of Natural Sciences \\ University of Stirling Stirling UK ahsan.adeel@stir.ac.uk, ahu@cs.stir.ac.uk
}

\begin{abstract}
Data annotation is an important but time-consuming and costly procedure. To sort a text into two classes, the very first thing we need is a good annotation guideline, establishing what is required to qualify for each class. In the literature, the difficulties associated with an appropriate data annotation has been underestimated. In this paper, we present a novel approach to automatically construct an annotated sentiment corpus for Algerian dialect (A Maghrebi Arabic dialect). The construction of this corpus is based on an Algerian sentiment lexicon that is also constructed automatically. The presented work deals with the two widely used scripts on Arabic social media: Arabic and Arabizi. The proposed approach automatically constructs a sentiment corpus containing 8000 messages (where 4000 are dedicated to Arabic and 4000 to Arabizi). The achieved F1-score is up to $72 \%$ and $78 \%$ for an Arabic and Arabizi test sets, respectively. Ongoing work is aimed at integrating transliteration process for Arabizi messages to further improve the obtained results.
\end{abstract}

Keywords: Arabic sentiment analysis, Algerian dialect, sentiment lexicon, sentiment corpus, sentiment classification

\section{Introduction}

Sentiment analysis is defined as an interdisciplinary domain among the natural language processing (NLP), artificial intelligence (AI), and text mining [17]. To determine whether a document or a sentence expresses a positive or negative sentiment, three main approaches are commonly used: the lexicon based approach [26], machine learning (ML) based approach [20] and a hybrid approach [18]. English has the greatest number of sentiment analysis studies, while research is more limited for other languages including Arabic and its dialects [5].

ML based sentiment analysis requires an annotated data. The lexicon based approach needs an annotated sentiment lexicon (containing the valence and/or intensity of its terms and/or expressions). One of the majors problems related 
to the treatment of Arabic and its dialect is the lack of resources. Other dominant problems include the standard romanization (called Arabizi) that Arabic speakers often use in social media. Arabizi uses Latin alphabet, numbers, punctuation for writing an Arabic word (For example the word "mli7", combining between Latin letters and numbers, is the romanized form of the Arabic word "مليح" meaning "good"). To the best of our knowledge, limited work has been conducted on sentiment analysis of Arabizi and it is dedicated to Arabic and not to its dialect [11. However, not much work has been conducted on sentiment analysis of Algerian Arabizi.

To bridge the gap, this paper proposes an approach that automatically construct a sentiment lexicon for a Magheribi dialect (i.e. Algerian dialect). Based on the constructed lexicon, we automatically annotate a sentiment corpus into positive and negative. To validate the build corpus, we applied a set of classifiers and tested our corpus on two different test sets: internal (which is a part of the constructed corpus) and external (which represent a set of messages that we manually annotated). However, the general experimental results have shown better performance with Arabic test sets which is attributed to the complexity of Arabizi.

This paper is organized as following: Section 2 presents the related work on sentiment analysis by focusing on the work done on Arabic and its dialects. Section 3 presents our approach and the different parts that composed it. Section 4 presents the different results that we collected in this study. Section 5 presents conclusion containing some opening for our futures works.

\section{Arabic Sentiment Analysis}

\subsection{Lexicon-based approaches}

A lexicon of 120,000 Arabic terms is build in [3, following infinitives collection, transliteration to English, and exploitation of English lexicon to determine the valence and intensity of each word. Another large lexicon has been constructed in [10]. It contains 157969 synonymous and 28760 lemmas. To build this big dataset, the authors combined several Arabic resources including English WordNet, Arabic WordNet, English SentiWordNet, Standard Arabic Morphological Analyzer (SAMA) . In [24, the authors develop a lexicon of sentiment containing 14182 English unigram classified into positive or negative using the "Mechanical Turk of Amazon" 4 . This lexicon is then translated into 40 languages including MSA. The authors in [2] studied three lexical construction techniques including one manual and two automatics. In addition, a SA tool was developed within this work. Experiments showed that the use of a lexicon containing 16800 words (created by integrating three techniques, so one manual and the two others automatic) gives the best results. In [21], the authors manually construct a lexicon of sentiment starting with an existing Arabic and Egyptian lexicon. They analyzed messages containing MSA as well as DALG. To answer to the morphological

\footnotetext{
${ }^{4}$ https://www.mturk.com/
} 
characteristics of this language and dialect, the authors used the lemmatization tool "khoja" (developed for MSA).

Most of the proposed lexicon construction methods are based on three: 1) manual; 2) automatic translation and 3) annotated corpus.In this paper, we exploited the second technique to construct our Algerian sentiment lexicon.

\subsection{Machine learning based approaches}

Supervised approaches essentially depends on the existence of annotated data. Among the corpora presented in the literature, we cite: OCA [25], AWATIF [1],LABR [9], TSAC [22], AraSenTi-Tweet 4]. OCA contains 500 Arabic comments (250 positive and 250 negative), manually preprocessed, then segmented, and root extracted with a tool dedicated to Arabic. AWATIF is a multi-genre corpus containing 10723 sentences in Arabic manually annotated in objective and subjective sentences. Then annotation of the subjective sentences in positive, negative or neutral. LABR contains 63257 Arabic comments annotated with stars ranging from 1 to 5 by users. The authors considered positive comments containing 4 or 5 stars, negative ones containing 1 or 2 stars and neutral ones containing 3 stars. TSAC contains 17060 comments (including 8215 positive and 8845 negative) in Tunisian dialect annotated manually. AraSenTi-Tweet contains 17573 Saudi tweets, manually annotated into four classes (positive, negative, neutral and mixed).

Almost all works are based on the constructed corpus to classify sentiment (by using classification algorithm). The most used classification Algorithm are: Support Vector Machine (SVM) and Naive Bays(NB). However, most of the aforementioned works suffer from: manual annotation, almost all resources are not publicly available and constructed corpora are not dedicated to DALG. To the best of our knowledge, one work only has been done on Arabizi sentiment analysis [11. However, it is not focus on Algerian Arabizi. Emergent works have been done on Algerian Arabizi treatment [12|16|14|13|15] but no one concentrate on sentiment analysis

\section{Contribution}

In this paper, we present an approach for sentiment analysis of DALG messages. This approach is based on an annotated corpus that we constructed automatically. The construction of this corpus is based on a sentiment lexicon (that we also constructed automatically) and on a sentiment algorithm (handling DALG characteristics). Figure 1 presents the proposed sentiment analysis approach. The proposed approach constitutes three general steps: 1) Automatic construction of DALG sentiment lexicon. 2) Polarity calculation of DALG messages and 3) Sentiment classification of DALG messages. The three steps are comprehensively explained in subsequent sections. 


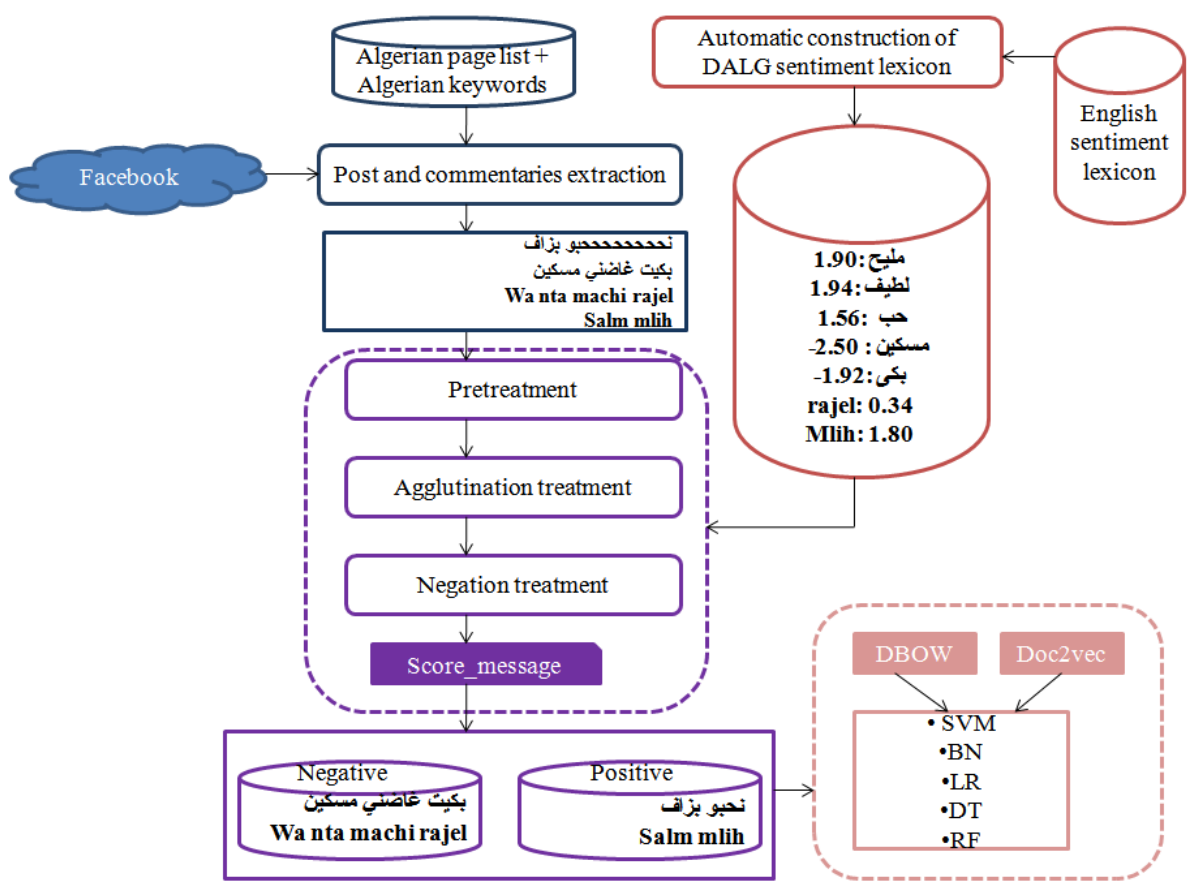

Fig. 1. A general architecture of our approach for sentiment analysis DALG

\subsection{Automatic construction of DALG sentiment lexicon}

Our approach receives a lexicon of sentiments in English as input. Each word in this lexicon is translated using a translation API (5). The specificity of this API is that the translation is performed by ordinary users native of the DALG. The same score is assigned to all collected words. For example, the English word 'excellent' (with a score of +5 ) gives different DALG words such as: 'بَاهي' baAhiy, 'مليح', mliyH, etc. All these words receive a score of +5 , similarly to the word 'excellent'. However, a word in DALG is associated with several words in English and can therefore have several scores. For example the word مليح mliyH, 'good' is associated with several English words such as: excellent, generous, delicious, etc. The word 'excellent' has a score equal to $(+5)$; the word 'generous' has the score $(+2)$ and the others words have different scores. Therefore we extract, within this part, all the words in DALG and calculating their scores. Concerning the calculation of the score, we take the average of the scores of all the English words to which our word in DALG is associated.

\footnotetext{
${ }^{5}$ https://glosbe.com/en/arq/excellent
} 


\subsection{Polarity calculation of DALG messages}

The goal of this step is to automatically annotate a set of Facebook messages (extracted from Algerian pages) as positive and negative. For example, the sentence: 'نحبو بزًاف' translated into 'I love him a lot' should recognized as positive and the sentence 'wa nta machi rajel' translated into 'and you are not man' should recognized as negative. To correctly annotate this sentences and others, we need to proceed to a set of treatments: 1) Pretreatment of the messages. 2) Agglutination treatment. 3) Negation treatment.

\subsubsection{Pretreatment of the messages}

- Deleting of the repeated messages in the corpus to keep only one occurrence of each message.

- Deleting of exaggerations, for example the word نحّحّحّحب is transformed into: نحب and nhhhhhab is transformed into nhab. The different repetitions of the different letter ' $C$ ' and 'h' are removed to keep a single occurrence.

- Deleting of the '\#' character and spaces of the different punctuations '.,!,?' of the related word .

- Deleting of consecutive whites spaces as well as Tatweel ('-') within Arabic characters.

3.2.2 Agglutination treatment We first form all the possible n-gram in the messages. For example, the second message ('بكيت غَاظني مسكين') gives us 3 uni-gram ('مسكين aكيت، غَاظني'), 2 bi-gram (بكيت غَاظني، غَاظني مسكين') and one tri-gram ('بكيت غَاظني مسكيني'). Then, we look for each n-gram in the lexicon and add the score of the finding ones into the global score (if they are not proceeded by a negation). If no n-gram is found, we extract stem from each word and look for it in the lexicon. To extract the DALG stems, we define a set of prefixes and suffixes (Personnel pronouns, complement pronouns, feminine pronouns, plural pronouns, etc) of DALG. For example: The stem of the word

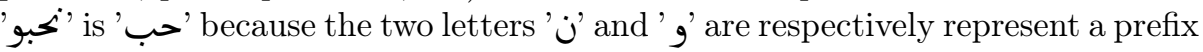

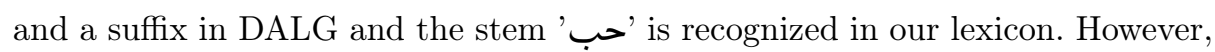
some words which are conjugated into the past like 'بكيت' to be transformed before being recognized. So, the stem of 'بكيت' is 'بكى'. To recognize it, we have to extract the part without affixes (so 'بك' and add the letter ' $ى$ ' at the end).

3.2.3 Negation treatment Negation analysis is an important research challenge for all languages. Nevertheless this challenge is accentuated in the case of Arabic and its dialects where the negation is usually attached to the word 
as well as the different pronouns. Users can use negation in different ways, for example the word مَانحبكمش can be written in the following way: مَا نحبكمش or

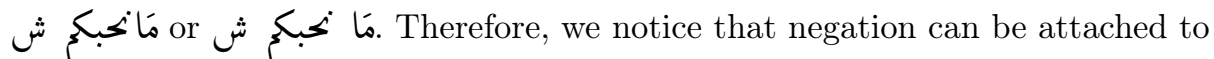
or separated from terms. We have found, however, that in most cases negation does not only affect the preceding word but also some other words in the sentence. Once a prefix or negation suffix is detected, we reverse the score of the words succeeding this negation (multiplying the score by $(-1)$ ).

After calculating the score of each messages, we annotate it as positive (if its score is bigger than 0), and negative in the other case (so if its score is smaller that 0 ). Finally, we are able to automatically annotate the extracted corpus.

\subsection{Sentiment classification of DALG messages}

In this paper, we propose to compare different shallow classification models. We used two vectorization techniques: Bag of Words (BOW) and document embedding where we rely on the Doc2vec algorithm presented within [19]. For Doc2vec, we apply the two methods presented in [19: 1) Distributed Memory Version Of Paragraph Vector (PV-DM) et 2) Distributed Bag of Words Version of Paragraph Vector (PV-DBOW). We also use the implementation merging these two methods. In the method (PV-DM), the paragraph vector (document or sentence) is concatenated to the word vectors in order to predict the next word within a text window. Unlike this method, (PV-BOW) ignores the context of the words within the inputs and this to force the prediction of these words randomly focusing on the paragraph vector. For the classification part, we use different classifiers: 1) Support Vector Machine (SVM). 2) Naive Bayes (NB). 3) Logistic regression (LR). 4) Decision Tree (DT) and 5) Random Forest (RF).

\section{Experimentations and results}

\subsection{Constructed resources}

In this work we constructed three kind of resources: 1) An Algerian sentiment lexicon (containing words in both Arabic and Arabizi). 2) A monolingual Algerian dialect corpus. 3) An annotated Algerian sentiment corpus.

For the construction of lexicons, we used SOCAL (an English sentiment lexicon) presented in [26]. SOCAL contains 6769 terms whose sentiment is labeled between $(-1,-5)$ for negative terms and between $(+1,+5)$ for positive terms. However only 3968 terms in English have been recognized and translated by the Glosbe API. After the extraction of Algerian dialect terms we obtain a lexicon containing 4873 words annotated from $(-5$ to +5$)$, where 2390 are in Arabic and 2483 are in Arabizi.

We use Socialbekers websit 6 to collect the name of the 226 most famous Algerian Facebook pages including Ooredoo, HamoudBoualem, Algeria Telecom,

\footnotetext{
${ }^{6}$ https://www.socialbakers.com/statistics/facebook/pages/total/algeria/
} 
Ruiba, etc. We also extracted some strong dialectal Algerian keywords from PADIC corpus[23] (for example, يفرح which means "he is happy", الكًَاب which means "a lier") .Then, we use RestFB ${ }^{7}$ API implemented with JAVA to extract all post and commentaries present in the target pages and present in others pages but containing Algerian keywords. At the end, we were able to collect $\mathbf{1 5 , 4 0 7 , 9 1 0}$ messages where $\mathbf{7 , 9 2 6 , 5 0 4}$ are in Arabic and $\mathbf{3 , 9 7 6 , 7 0 0}$ are in Arabizi.

Based on the constructed lexicon, on the extracted corpus and on the sentiment analysis algorithm that we developed, we are able to automatically annotate a sentiment corpus. Then we randomly form a training corpus containing 8000 messages (where 4000 messages are in Arabic and 4000 messages are in Arabizi with 2000 positives messages and 2000 negative. After, this corpus is divided into three part (train, dev and test) for internal experiment and (train, dev) for external experiment. The dev set is used only for deep classification. For shallow classification, we use the entire annotated corpus as training.

\subsection{Experimental results}

The constructed automatically annotated sentiment corpus has been critically analyzed by applying shallow algorithms. Difficulties to deal with a non-resourced dialect (such as Algerian dialect which uses two different alphabets) have also been highlighted.Table 1 presents the performance of difference classification algorithms in terms of Accuracy (Acc)) and F1-score (F1) for each vectorization method (BOW, Doc2vec).

Based on simulations and analysis, our three major observations are: 1) The results with Arabic sets are better than Arabizi sets in most of the cases. 2) The results on internal data sets are better than external ones. 3) Bow vectorization gives better results than Doc2vec. These results are principally related to five facts and could substantially be improved by handling these lack:

- Arabizi is very complex and one term could have different writing manner (sometimes more than 100 manners). handling it with one lexicon is almost impossible. To address this issues, we could enrich the used lexicon by all variation of Arabizi word. We also propose to integrate a transliteration module to transform Arabizi into Arabic before analyzing the sentiment of Arabizi messages.

" بur lexicon contains lots of noise. For example the different words: 1) " و ، مع (2) (12) etc (with) have a negative polarity when these words are neural.2) The word (رب" meaning "god" has a positive polarity in Arabic when the word "lah" representing a part of "alah" in Arabizi (meaning god too) has a negative polarity. To address this problem, a manual review of lexicons could be used to increase the precision of annotation.

\footnotetext{
7 http://restfb.com/
} 


\begin{tabular}{|c|c|c|c|c|c|c|c|c|c|c|}
\hline \multirow{3}{*}{\multicolumn{2}{|c|}{ Vectorization }} & \multirow{3}{*}{ Classification } & \multicolumn{4}{|c|}{ Arabic } & \multicolumn{4}{|c|}{ Arabizi } \\
\hline & & & \multicolumn{2}{|c|}{ Internal } & \multicolumn{2}{|c|}{ External } & \multicolumn{2}{|c|}{ Internal } & \multicolumn{2}{|c|}{ External } \\
\hline & & & F1 & Acc & F1 & Acc & F1 & Acc & F1 & Acc \\
\hline \multirow{5}{*}{\multicolumn{2}{|c|}{ Bow }} & SVM & 0.51 & 0.54 & 0.40 & 0.47 & 0.57 & 0.61 & 0.42 & 0.45 \\
\hline & & NB & 0.56 & 0.57 & 0.51 & 0.55 & 0.56 & 0.58 & 0.55 & 0.57 \\
\hline & & LR & 0.72 & 0.72 & 0.68 & 0.68 & 0.78 & 0.78 & 0.51 & 0.52 \\
\hline & & DT & 0.67 & 0.67 & 0.58 & 0.59 & 0.75 & 0.75 & 0.49 & 0.49 \\
\hline & & RF & 0.67 & 0.67 & 0.59 & 0.61 & 0.73 & 0.73 & 0.49 & 0.49 \\
\hline \multirow{15}{*}{ Doc2vec } & \multirow{5}{*}{ PV-DBOW } & SVM & 0.57 & 0.58 & 0.63 & 0.63 & 0.51 & 0.54 & 0.61 & 0.61 \\
\hline & & NB & 0.52 & 0.52 & 0.67 & 0.67 & 0.52 & 0.52 & 0.59 & 0.60 \\
\hline & & LR & 0.59 & 0.59 & 0.63 & 0.64 & 0.54 & 0.54 & 0.53 & 0.55 \\
\hline & & DT & 0.55 & 0.56 & 0.58 & 0.58 & 0.54 & 0.54 & 0.53 & 0.53 \\
\hline & & RF & 0.58 & 0.59 & 0.61 & 0.61 & 0.55 & 0.56 & 0.55 & 0.56 \\
\hline & \multirow{5}{*}{ PV-DM } & SVM & 0.48 & 0.52 & 0.51 & 0.54 & 0.50 & 0.52 & 0.61 & 0.62 \\
\hline & & NB & 0.41 & 0.48 & 0.45 & 0.53 & 0.52 & 0.52 & 0.65 & 0.65 \\
\hline & & LR & 0.53 & 0.53 & 0.57 & 0.58 & 0.52 & 0.52 & 0.63 & 0.63 \\
\hline & & DT & 0.54 & 0.54 & 0.56 & 0.56 & 0.51 & 0.51 & 0.49 & 0.49 \\
\hline & & RF & 0.52 & 0.53 & 0.53 & 0.54 & 0.52 & 0.53 & 0.51 & 0.51 \\
\hline & \multirow{5}{*}{$\begin{array}{l}\text { PV-DBOW/ } \\
\text { PV-DM }\end{array}$} & SVM & 0.44 & 0.49 & 0.51 & 0.53 & 0.51 & 0.54 & 0.61 & 0.63 \\
\hline & & NB & 0.41 & 0.48 & 0.48 & 0.55 & 0.51 & 0.51 & 0.66 & 0.66 \\
\hline & & LR & 0.51 & 0.51 & 0.55 & 0.56 & 0.54 & 0.54 & 0.66 & 0.66 \\
\hline & & DT & 0.53 & 0.53 & 0.45 & 0.45 & 0.49 & 0.49 & 0.55 & 0.55 \\
\hline & & RF & 0.46 & 0.47 & 0.53 & 0.54 & 0.49 & 0.50 & 0.55 & 0.56 \\
\hline
\end{tabular}

Table 1. DALG Sentiment analysis results

- Other parameters than score involved in the annotation process such as: number of positives and negatives words, length of the sentence, the comparison of score to other threshold than 0 , etc. The integration of these parameters to our algorithm and testing and the influence of each one on the annotation process could considerably improve t,he results.

- Some irregular plurals such as 2 in arabic and "mlah" in Arabizi are not recognize by our algorithm which is only based on soft stemming. The proposition of a stemmer tool dedicated to DALG could improve the annotation process.

- The vectorization used techniques are complementary. Hence, some messages are recognized by using DBOW and are not recognized by using Doc2vec and vice versa. The combination between the different vectorization technique will considerably improve the results.

\section{Conclusion and Perspectives}

In this paper, we present a novel approach to automatically construct an annotated sentiment corpus for Algerian dialect (A Maghrebi Arabic dialect). The construction of this corpus is based on an Algerian sentiment lexicon that is also constructed automatically. The presented work deals with the two widely used scripts on Arabic social media: Arabic and Arabizi. The 
proposed approach automatically constructs a sentiment corpus containing 8000 messages (where 4000 are dedicated to Arabic and 4000 to Arabizi). The achieved F1-score is up to $72 \%$ and $78 \%$ for an Arabic and Arabizi internal test sets, and up to $68 \%$ and $66 \%$ for an Arabic and Arabizi internal test sets respectively.

This study represents the baseline for our future work where we plan to augmenting the lexicon size based on embedding algorithm such as word2vec. Additionally, enlarging the dataset with focusing on more annotated words will provide much better results. One thing more, one arabizi word could have many different writing manners. This phenomena leads to misinterpretation and consequently a wrong polarity classification. Hence, proceeding to transliterate arabizi messages absolutely improve the results.

We also plan to extend our work for handling handwriting Arabic based on the work proposed in 8776 .

\section{Acknowledgment}

Amir Hussain and Ahsan Adeel were supported by the UK Engineering and Physical Sciences Research Council (EPSRC) grant No.EP/M026981/1.

\section{References}

1. Abdul-Mageed, M., Diab, M.T.: Awatif: A multi-genre corpus for modern standard arabic subjectivity and sentiment analysis. In: LREC. pp. 3907-3914. Citeseer (2012)

2. Abdulla, N., Mohammed, S., Al-Ayyoub, M., Al-Kabi, M., et al.: Automatic lexicon construction for arabic sentiment analysis. In: Future Internet of Things and Cloud (FiCloud), 2014 International Conference on. pp. 547-552. IEEE (2014)

3. Al-Ayyoub, M., Essa, S.B., Alsmadi, I.: Lexicon-based sentiment analysis of arabic tweets. International Journal of Social Network Mining 2(2), 101-114 (2015)

4. Al-Twairesh, N., Al-Khalifa, H., Al-Salman, A., Al-Ohali, Y.: Arasenti-tweet: A corpus for arabic sentiment analysis of saudi tweets. Procedia Computer Science 117, 63-72 (2017)

5. Alayba, A.M., Palade, V., England, M., Iqbal, R.: Arabic language sentiment analysis on health services. In: Arabic Script Analysis and Recognition (ASAR), 2017 1st International Workshop on. pp. 114-118. IEEE (2017)

6. AlKhateeb, J.H., Jiang, J., Ren, J., Ipson, S.: Component-based segmentation of words from handwritten arabic text. International Journal of Computer Systems Science and Engineering 5(1) (2009)

7. AlKhateeb, J.H., Pauplin, O., Ren, J., Jiang, J.: Performance of hidden markov model and dynamic bayesian network classifiers on handwritten arabic word recognition. knowledge-based systems 24(5), 680-688 (2011)

8. AlKhateeb, J.H., Ren, J., Jiang, J., Al-Muhtaseb, H.: Offline handwritten arabic cursive text recognition using hidden markov models and re-ranking. Pattern Recognition Letters 32(8), 1081-1088 (2011)

9. Aly, M., Atiya, A.: Labr: A large scale arabic book reviews dataset. In: Proceedings of the 51st Annual Meeting of the Association for Computational Linguistics (Volume 2: Short Papers). vol. 2, pp. 494-498 (2013) 
10. Badaro, G., Baly, R., Hajj, H., Habash, N., El-Hajj, W.: A large scale arabic sentiment lexicon for arabic opinion mining. In: Proceedings of the EMNLP 2014 Workshop on Arabic Natural Language Processing (ANLP). pp. 165-173 (2014)

11. Duwairi, R.M., Alfaqeh, M., Wardat, M., Alrabadi, A.: Sentiment analysis for arabizi text. In: Information and Communication Systems (ICICS), 2016 7th International Conference on. pp. 127-132. IEEE (2016)

12. GUELliL, I., AZOUAOU, F.: Bilingual lexicon for algerian arabic dialect treatment in social media (2017)

13. Guellil, I., Azouaou, F., Abbas, M.: Comparison between neural and statistical translation after transliteration of algerian arabic dialect. In: WiNLP: Women \& Underrepresented Minorities in Natural Language Processing (co-located withACL 2017). pp. 1-5 (2017)

14. Guellil, I., Azouaou, F., Abbas, M., Fatiha, S.: Arabizi transliteration of algerian arabic dialect into modern standard arabic. In: Social MT 2017/First workshop on Social Media and User Generated Content Machine Translation. pp. 1-8 (2017)

15. Guellil, I., Azouaou, F.: Arabic dialect identification with an unsupervised learning (based on a lexicon). application case: Algerian dialect. In: Computational Science and Engineering (CSE) and IEEE Intl Conference on Embedded and Ubiquitous Computing (EUC) and 15th Intl Symposium on Distributed Computing and Applications for Business Engineering (DCABES), 2016 IEEE Intl Conference on. pp. 724-731. IEEE (2016)

16. Guellil, I., Azouaou, F.: Asda: Analyseur syntaxique du dialecte alg $\{\backslash$ 'e $\}$ rien dans un but d'analyse s $\{\backslash$ 'e\} mantique pp. 87-94 (2017)

17. Guellil, I., Boukhalfa, K.: Social big data mining: A survey focused on opinion mining and sentiments analysis. In: Programming and Systems (ISPS), 2015 12th International Symposium on. pp. 1-10. IEEE (2015)

18. Khan, A.Z., Atique, M., Thakare, V.: Combining lexicon-based and learning-based methods for twitter sentiment analysis. International Journal of Electronics, Communication and Soft Computing Science \& Engineering (IJECSCSE) p. 89 (2015)

19. Le, Q., Mikolov, T.: Distributed representations of sentences and documents. In: International Conference on Machine Learning. pp. 1188-1196 (2014)

20. Maas, A.L., Daly, R.E., Pham, P.T., Huang, D., Ng, A.Y., Potts, C.: Learning word vectors for sentiment analysis. In: Proceedings of the 49th annual meeting of the association for computational linguistics: Human language technologies-volume 1. pp. 142-150. Association for Computational Linguistics (2011)

21. Mataoui, M., Zelmati, O., Boumechache, M.: A proposed lexicon-based sentiment analysis approach for the vernacular algerian arabic. Research in Computing Science 110, 55-70 (2016)

22. Medhaffar, S., Bougares, F., Esteve, Y., Hadrich-Belguith, L.: Sentiment analysis of tunisian dialects: Linguistic ressources and experiments. In: Proceedings of the Third Arabic Natural Language Processing Workshop. pp. 55-61 (2017)

23. Meftouh, K., Harrat, S., Jamoussi, S., Abbas, M., Smaili, K.: Machine translation experiments on padic: A parallel arabic dialect corpus. In: The 29th Pacific Asia conference on language, information and computation. pp. 1-9 (2015)

24. Mohammad, S.M., Turney, P.D.: Crowdsourcing a word-emotion association lexicon. Computational Intelligence 29(3), 436-465 (2013)

25. Rushdi-Saleh, M., Martín-Valdivia, M.T., Ureña-López, L.A., Perea-Ortega, J.M.: Oca: Opinion corpus for arabic. Journal of the Association for Information Science and Technology 62(10), 2045-2054 (2011)

26. Taboada, M., Brooke, J., Tofiloski, M., Voll, K., Stede, M.: Lexicon-based methods for sentiment analysis. Computational linguistics 37(2), 267-307 (2011) 